\title{
Ground state structures and properties of small hydrogenated silicon clusters
}

\author{
R PRASAD \\ Department of Physics, Indian Institute of Technology, Kanpur 208 016, India
}

\begin{abstract}
We present results for ground state structures and properties of small hydrogenated silicon clusters using the Car-Parrinello molecular dynamics with simulated annealing. We discuss the nature of bonding of hydrogen in these clusters. We find that hydrogen can form a bridge like Si-H-Si bond connecting two silicon atoms. We find that in the case of a compact and closed silicon cluster hydrogen bonds to the silicon cluster from outside. To understand the structural evolutions and properties of silicon cluster due to hydrogenation, we have studied the cohesive energy and first excited electronic level gap of clusters as a function of hydrogenation. We find that first excited electronic level gap of $\mathrm{Si}_{n}$ and $\mathrm{Si}_{n} \mathrm{H}$ fluctuates as function of size and this may provide a first principle basis for the short-range potential fluctuations in hydrogenated amorphous silicon. The stability of hydrogenated silicon clusters is also discussed.
\end{abstract}

Keywords. Ground state; hydrogenated silicon; Car-Parrinello; simulated annealing.

\section{Introduction}

During the last decade there has been a very rapid development in the study of small clusters. This has been partly due to the growing importance of these systems in applications like catalysis and due to possibilities of developing nano-electronic devices. Also some clusters are found to be very stable and referred to as magic clusters. There have been attempts to design novel materials using these clusters as basic building blocks. These materials may have very different properties compared to the naturally occurring solids.

Small hydrogenated silicon clusters are of particular interest because of the importance of the materials like hydrogenated amorphous silicon $(a-\mathrm{Si}: \mathrm{H})$ and porous silicon. These materials are important, not only from technological point of view, they also exhibit interesting phenomena like Staebler-Wronski effect $(a-\mathrm{Si}: \mathrm{H})$ and photoluminescence (porous silicon) which are not well understood. These systems are difficult to model theoretically as these are highly disordered. However, if one is looking at some generic features such as changes in structure or electronic structure due to addition of hydrogen, some understanding can be gained by studying small clusters of silicon and hydrogen, which are much easier to model. This has been the prime motivation behind this study.

We have carried out our study using the Car-Parrinello molecular dynamics. This is an ab initio method which involves no adjustable or empirical parameter. In $\S 2$, we briefly discuss the Car-Parrinello method. In $\S 3$, we dis-

\footnotetext{
*Author for correspondence
}

cuss the ground state structures of small hydrogenated clusters and in $\$ 4$, we discuss how the stability of silicon cluster is affected by hydrogenation. Finally in $\$ 5$ we give our main conclusions and their implications for $a-\mathrm{Si}: \mathrm{H}$.

\section{Car-Parrinello method}

Car and Parrinello (1985) proposed a Lagrangian in which the single particle electronic orbitals, $\psi(\vec{r}, t)$, are also dynamical variables along with nuclear coordinates, $\vec{R}(t)$. The dynamics of nuclei is real while the dynamics of the orbitals is fictitious. This was a great idea which led to considerable saving in computational cost and made the first-principle molecular dynamics possible. They proposed the following Lagrangian

$$
\begin{aligned}
& L=\frac{1}{2} \sum_{\ell} M\left|\dot{\vec{R}}_{\ell}\right|^{2}+\sum_{i, \mathrm{occ}} \int \mu\left|\dot{\Psi}_{i}(\vec{r}, t)\right|^{2} \mathrm{~d}^{3} r \\
& -E\left[\left\{\Psi_{i}\right\}\left\{\vec{R}_{\ell}\right\}\right]+\sum_{i j} \Lambda_{i j}\left(\int \Psi_{i}^{*}(\vec{r}, t) \Psi_{j}(\vec{r}, t) \mathrm{d}^{3} r-\delta_{i j}\right)
\end{aligned}
$$

where

$$
\begin{aligned}
& E\left[\left\{\psi_{i}\right\}\left\{\vec{R}_{\ell}\right\}\right]=E_{E}(\vec{R})+V_{\mathrm{II}}(\vec{R}) \\
&= \sum_{i=1}^{N} \int \Psi_{i}^{*}(\vec{r}, t)\left(-\frac{\hbar^{2}}{2 m} \nabla^{2}\right) \Psi_{i}(\vec{r}, t) \mathrm{d}^{3} r \\
&+\int v(\vec{r}) n(\vec{r}) \mathrm{d}^{3} r+\frac{1}{2} \iint \frac{n(\vec{r}) n\left(\vec{r}^{\prime}\right)}{\left|\vec{r}-\vec{r}^{\prime}\right|} \mathrm{d}^{3} r \mathrm{~d}^{3} r^{\prime}
\end{aligned}
$$




$$
+E_{\mathrm{xc}}[n]+V_{\mathrm{II}}(\vec{R}) .
$$

In (2) $V_{\mathrm{II}}(R)$ is the potential energy corresponding to ion-ion repulsion, $E_{\mathrm{xc}}[n]$ the exchange correlation energy and $n(\vec{r})$ the electron density given by

$$
n(\vec{r})=\sum_{\substack{i \\ \text { occ }}}\left|\psi_{i}(\vec{r}, t)\right|^{2} .
$$

The first term in (1) is the total kinetic energy of the atoms and the last term arises because of the constraint that the orbitals be orthonormal. In this term $\Lambda_{i j}$ are the Lagrange multipliers. Since the Lagrangian is real, the matrix $\Lambda$ is Hermitian, i.e. $\Lambda_{i j}=\Lambda_{j i}^{*}$. The second term was introduced by $\mathrm{Car}$ and Parrinello as a computational device and as such has no physical meaning. It represents a fictitious kinetic energy associated with the orbitals which are frequently referred to as electronic degrees of freedom. $\mu$ is fictitious mass such that in the limit $\mu \rightarrow 0$, we get the true Lagrangian of the system.

Using the Lagrangian (1), equations of motions for the dynamical variables $\left\{\vec{R}_{\ell}\right\}$ and $\left\{\psi_{i}\right\}$ can be written as

$$
M \ddot{\vec{R}}_{\ell}=-\frac{\partial E\left[\left\{\Psi_{i}\right\}\left\{\vec{R}_{\ell}\right\}\right]}{\partial \vec{R}_{\ell}}
$$

and

$$
\begin{aligned}
\mu \ddot{\psi}_{i}(\vec{r}, t) & =-\frac{\delta E\left[\left\{\Psi_{i}\right\}\left\{\vec{R}_{\ell}\right\}\right]}{\delta \Psi_{i}^{*}(\vec{r}, t)}+\sum_{j} \Lambda_{i j} \Psi_{j}(\vec{r}, t) \\
& =-H_{\mathrm{KS}} \Psi_{i}(\vec{r}, t)+\sum_{j} \Lambda_{i j} \Psi_{j}(\vec{r}, t),
\end{aligned}
$$

where $H_{\mathrm{KS}}$ is the Kohn-Sham Hamiltonian given by

$$
H_{\mathrm{KS}}=-\frac{\hbar^{2}}{2 m} \nabla^{2}+v(\vec{r})+v_{\mathrm{xc}}(\vec{r})+\int \frac{n\left(\vec{r}^{\prime}\right)}{\left|\vec{r}-\vec{r}^{\prime}\right|} \mathrm{d}^{3} r^{\prime} .
$$

Equations (4) and (6) are the desired equations of motion for $\left\{\vec{R}_{\ell}\right\}$ and $\left\{\psi_{i}(\vec{r}, t)\right\}$. If one starts from an initial condition, when all the electrons are in the ground state (i.e. the system is on B-O surface), from (4) and (5) one can find $\left\{\vec{R}_{\ell}(t)\right\}$ and $\left\{\psi_{i}(\vec{r}, t)\right\}$ by using say Verlet algorithm. Note that at subsequent time steps one need not solve the electronic structure problem but one simply updates the orbitals using (5). Thus the Car-Parrinello strategy results in tremendous saving in computational cost.

\section{Ground state structures}

The ground state structures were obtained by using the Car-Parrinello method and simulated annealing optimization. Wherever necessary we have also used steepest descent method. The details of the calculation are given elsewhere (Balamurugan and Prasad 2001).
The ground state geometries of $\mathrm{Si}_{n} \mathrm{H}$ clusters from $n=2$ to $n=6$ are shown in figure 1 . It is interesting to note that hydrogen is bonded with two silicon atoms in $\mathrm{Si}_{2} \mathrm{H}, \mathrm{Si}_{3} \mathrm{H}$ and $\mathrm{Si}_{5} \mathrm{H}$ although its valence is one. Such $\mathrm{Si}-$ $\mathrm{H}-\mathrm{Si}$ bridge type bonds are thought to be present in amorphous hydrogenated silicon and play an important role in explaining Staebler-Wronski effect. Two lowest energy structures of $\mathrm{Si}_{5} \mathrm{H}$ are very close in energy and differ only by $0.037 \mathrm{eV}$. We have shown both of these in figure 1. We find that although the addition of $\mathrm{H}$ does not affect the geometry of a silicon cluster much, it changes bond lengths and tries to distort the cluster.

In figure 2, we show the ground state structures of $\mathrm{Si}_{n} \mathrm{H}$ clusters from $n=7$ to $n=10$. In these structures, hydrogen is bonded to only one silicon atom in contrast to $\mathrm{Si}_{2} \mathrm{H}, \mathrm{Si}_{3} \mathrm{H}$ and $\mathrm{Si}_{5} \mathrm{H}$ clusters where it was bonded to two silicon atoms. From charge density calculations, we find that $\mathrm{Si}-\mathrm{H}$ and $\mathrm{Si}-\mathrm{H}-\mathrm{Si}$ bonds are polar covalent. We note that all these structures are closed and compact structures with hydrogen attached to a silicon atom from outside. From our results we find that hydrogen prefers to attach to an over-coordinated silicon atom. This is because hydrogen is slightly more electronegative than silicon and thus prefers a site where it finds more elec-

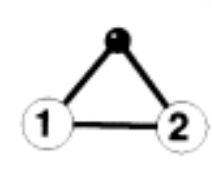

(a)

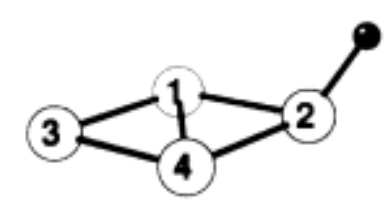

(c)

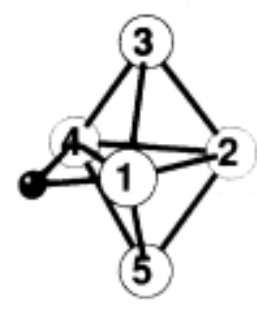

(e)

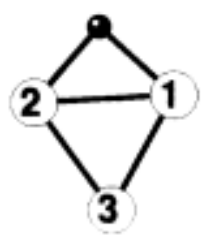

(b)

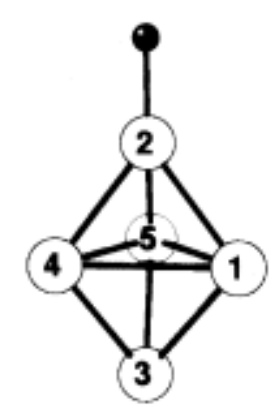

(d)

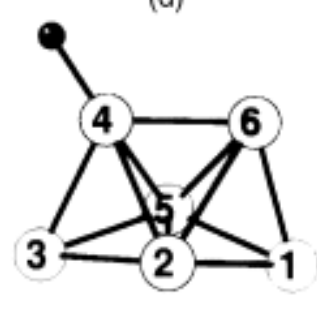

(f)
Figure 1. Ground state geometry of (a) $\mathrm{Si}_{2} \mathrm{H}$, (b) $\mathrm{Si}_{3} \mathrm{H}$, (c) $\mathrm{Si}_{4} \mathrm{H}$, (d) higher energy geometry of $\mathrm{Si}_{5} \mathrm{H}$, (e) ground state geometry of $\mathrm{Si}_{5} \mathrm{H}$ cluster and (f) ground state geometry of $\mathrm{Si}_{6} \mathrm{H}$ cluster. Silicon atoms are numbered and hydrogen atom is shown by a small dark circle. 
trons. Also, we find that hydrogen prefers to stay outside the compact structure. To examine this further, we performed simulations with hydrogen surrounded by silicon atoms. We found that hydrogen always comes out of the silicon cluster. This is because when hydrogen is inside a compact structure, the electrostatic energy is more compared to when it is outside.

The effect of adding more than one hydrogen on the geometry of $\mathrm{Si}_{2}$ cluster is shown in figure 3. The ground

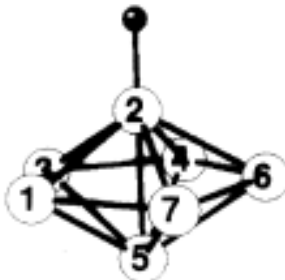

(a)

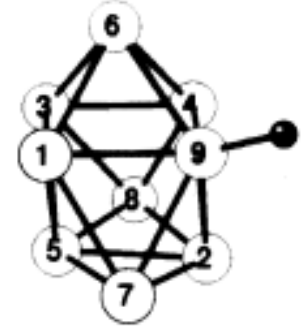

(c)

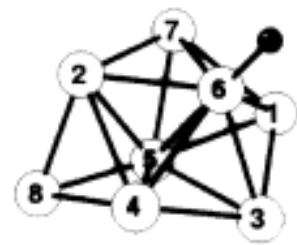

(b)

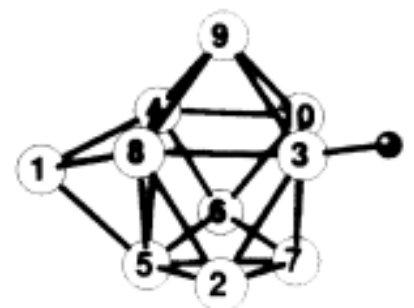

(d)
Figure 2. Ground state geometry of (a) $\mathrm{Si}_{7} \mathrm{H}$, (b) $\mathrm{Si}_{8} \mathrm{Hm}$, (c) $\mathrm{Si}_{9} \mathrm{H}$ cluster and (d) $\mathrm{Si}_{10} \mathrm{H}$ cluster.

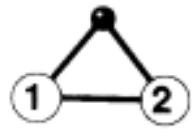

(a)

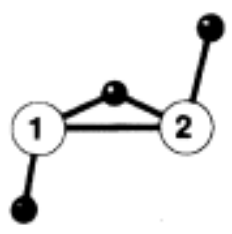

(c)

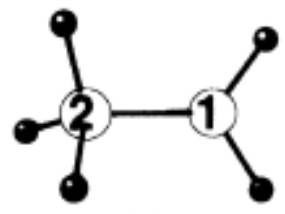

(e)

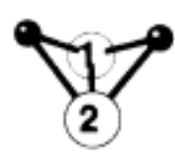

(b)

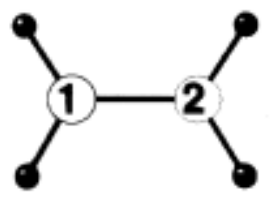

(d)

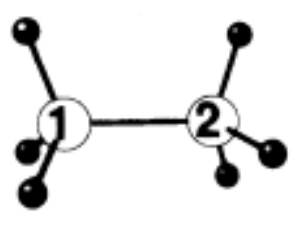

(f)
Figure 3. Ground state geometry of (a) $\mathrm{Si}_{2} \mathrm{H}$, (b) $\mathrm{Si}_{2} \mathrm{H}_{2}$, (c) $\mathrm{Si}_{2} \mathrm{H}_{3}$, (d) $\mathrm{Si}_{2} \mathrm{H}_{4}$, (e) $\mathrm{Si}_{2} \mathrm{H}_{5}$ and (f) $\mathrm{Si}_{2} \mathrm{H}_{6}$ cluster. Silicon atoms are numbered. state geometry of $\mathrm{Si}_{2} \mathrm{H}_{2}$ cluster is a non-planar rhombus, in which we see two $\mathrm{Si}-\mathrm{H}-\mathrm{Si}$ type bonds. This type of bond is also seen in $\mathrm{Si}_{2} \mathrm{H}_{3}$ whose ground state geometry is shown in figure 3(c). There is no $\mathrm{Si}-\mathrm{H}-\mathrm{Si}$ type bond in $\mathrm{Si}_{2} \mathrm{H}_{4}, \quad \mathrm{Si}_{2} \mathrm{H}_{5}$ and $\mathrm{Si}_{2} \mathrm{H}_{6}$ clusters. The variation of $\mathrm{Si}-\mathrm{Si}$ bond length in $\mathrm{Si}_{2} \mathrm{H}_{n}$ cluster as a function of $n$ is shown in figure 4. We see that hydrogenation can change the $\mathrm{Si}-$ Si bond length approximately by $10 \%$.

\section{Stability}

The first excited electronic level gap can be taken as a measure of the electronic stability of a system (Balamurugan and Prasad 2001). We have shown the first excited level gap as a function of cluster size in figure 5 for $\mathrm{Si}_{n} \mathrm{H}$ and $\mathrm{Si}_{n}$ clusters. Also shown in the figure are results of $\mathrm{Lu}$ et al (2000) for $\mathrm{Si}_{n}$ clusters which are in good agreement with our results. We see that the general trend of variation of the first excited electronic level gap is quite similar for $\mathrm{Si}_{n} \mathrm{H}$ clusters. The figure also shows that the addition of hydrogen can cause large changes in the electronic structure of $\mathrm{Si}_{n}$ cluster.

From figure 5 we see that the gap fluctuates with size, which indicates that the gap strongly depends on the size and geometry of a cluster. It might be interesting to draw parallels with short-range potential fluctuations in $a$ $\mathrm{Si}: \mathrm{H}$ system which occur at the length scale of $3 \AA$ (Fritzche 1971; Sinha and Agarwal 2000). It can be

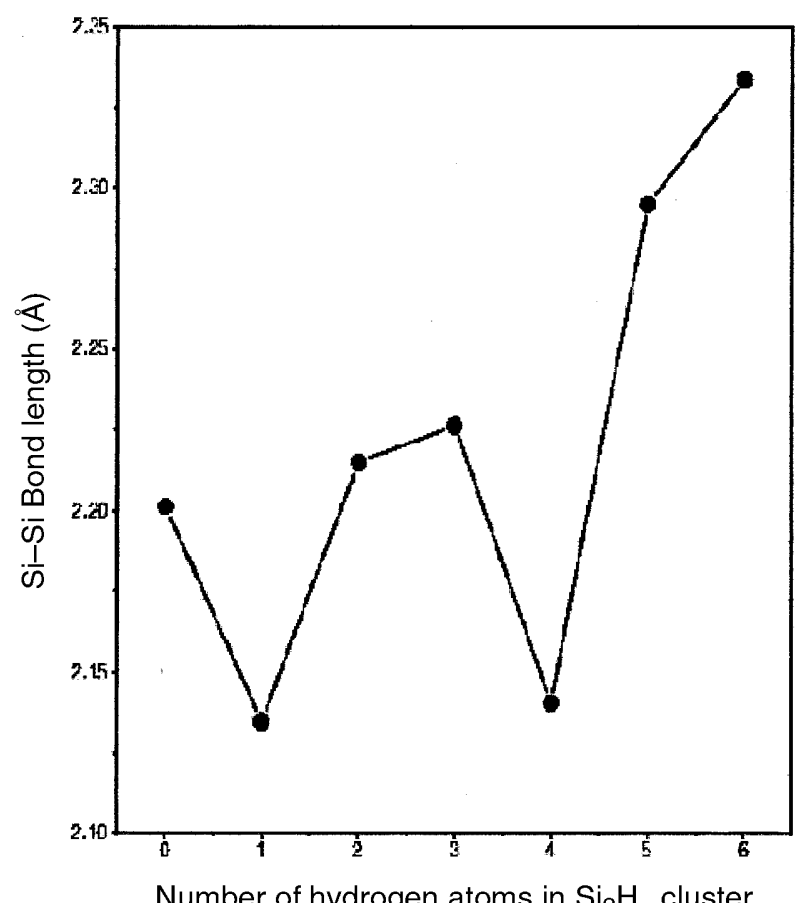

Figure 4. The $\mathrm{Si}-\mathrm{Si}$ bond of $\mathrm{Si}_{2} \mathrm{H}_{n}$ cluster vs number of hydrogen atoms in the cluster. 


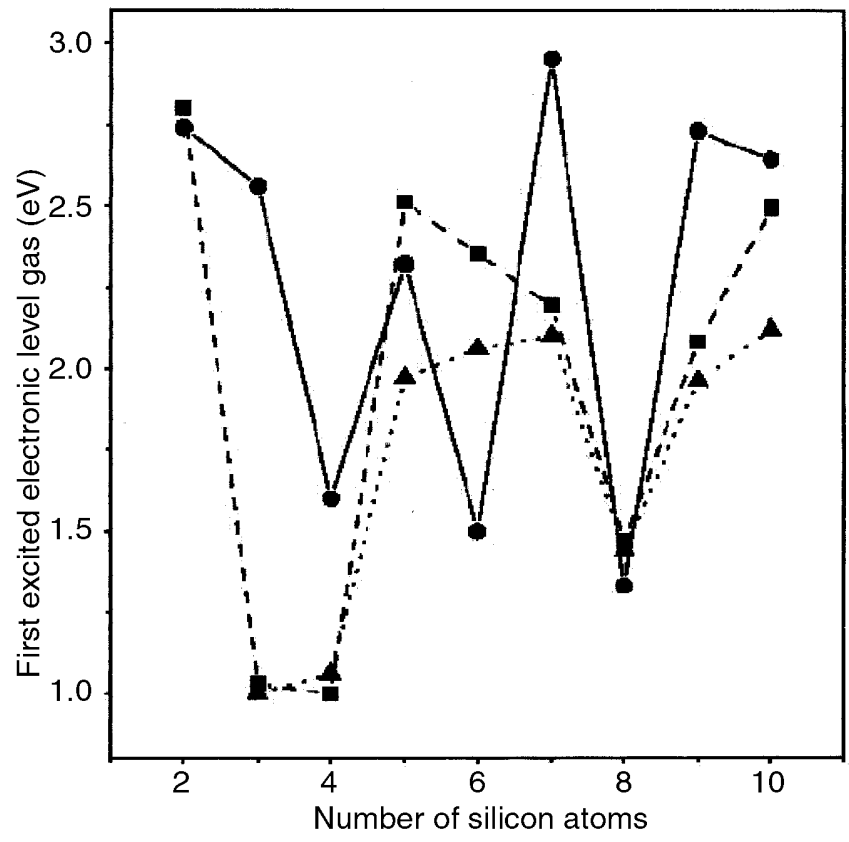

Figure 5. First excited state electronic level gap of $\mathrm{Si}_{n} \mathrm{H}$ and $\mathrm{Si}_{n}$ clusters vs number of silicon atoms in the cluster. The circles and squares correspond to $\mathrm{Si}_{n} \mathrm{H}$ and $\mathrm{Si}_{n}$ clusters, respectively. The triangles represent the results of $\mathrm{Lu}$ et al (2000) for $\mathrm{Si}_{n}$ cluster.

argued that an amorphous system can be considered as a loosely connected network of small clusters and thus our calculation provides a first-principle basis for the potential fluctuations (Fritzche 1971; Agarwal 1996a, b; Sinha and Agarwal 2000). Furthermore, we see that the first excited electronic level gap for $\mathrm{Si}_{n} \mathrm{H}$ is, on average, more than that of $\mathrm{Si}_{n}$ cluster. This is consistent with the observation that the band gap of $a: \mathrm{Si}$ increases on hydrogenation. Further, figure 5 shows that $\mathrm{Si}_{2} \mathrm{H}, \mathrm{Si}_{3} \mathrm{H}$, $\mathrm{Si}_{5} \mathrm{H}, \quad \mathrm{Si}_{7} \mathrm{H}, \quad \mathrm{Si}_{9} \mathrm{H}$ and $\mathrm{Si}_{10} \mathrm{H}$ clusters are electronically more stable compared to $\mathrm{Si}_{4} \mathrm{H}, \mathrm{Si}_{6} \mathrm{H}$ and $\mathrm{Si}_{8} \mathrm{H}$ clusters. Also we see that $\mathrm{Si}_{2}, \mathrm{Si}_{5}, \mathrm{Si}_{6}, \mathrm{Si}_{7}$ and $\mathrm{Si}_{10}$ clusters are electronically more stable than other silicon clusters $\left(\mathrm{Si}_{3}\right.$, $\mathrm{Si}_{4}, \mathrm{Si}_{8}$ and $\mathrm{Si}_{9}$ clusters), since they have larger gaps.

To examine geometrical stability we have also calculated the difference between the total energy of the modified $\mathrm{Si}_{n}$ cluster, which has the same positions of silicon atoms as in the $\mathrm{Si}_{n} \mathrm{H}$ cluster, and the ground state geometry of $\mathrm{Si}_{n}$ cluster. This energy difference is a measure of how much a silicon cluster distorts from its ground state geometry due to the addition of hydrogen atom. Lower value of this difference for a $\mathrm{Si}_{n}$ cluster means that the cluster is geometrically stable. This total energy difference as a function of cluster size, $n$, is shown in figure 6 . The figure shows that $\mathrm{Si}_{2}, \mathrm{Si}_{4}, \mathrm{Si}_{7}$ and $\mathrm{Si}_{10}$ clusters are geometrically more stable than $\mathrm{Si}_{3}, \mathrm{Si}_{5}, \mathrm{Si}_{6}$ and $\mathrm{Si}_{9}$ clusters. On the other hand, $\mathrm{Si}_{3}, \mathrm{Si}_{5}, \mathrm{Si}_{6}, \mathrm{Si}_{8}$ and $\mathrm{Si}_{9}$ clusters are stabilized by hydrogen and have a greater tendency to adsorb hydrogen. This is consistent with the conclusions

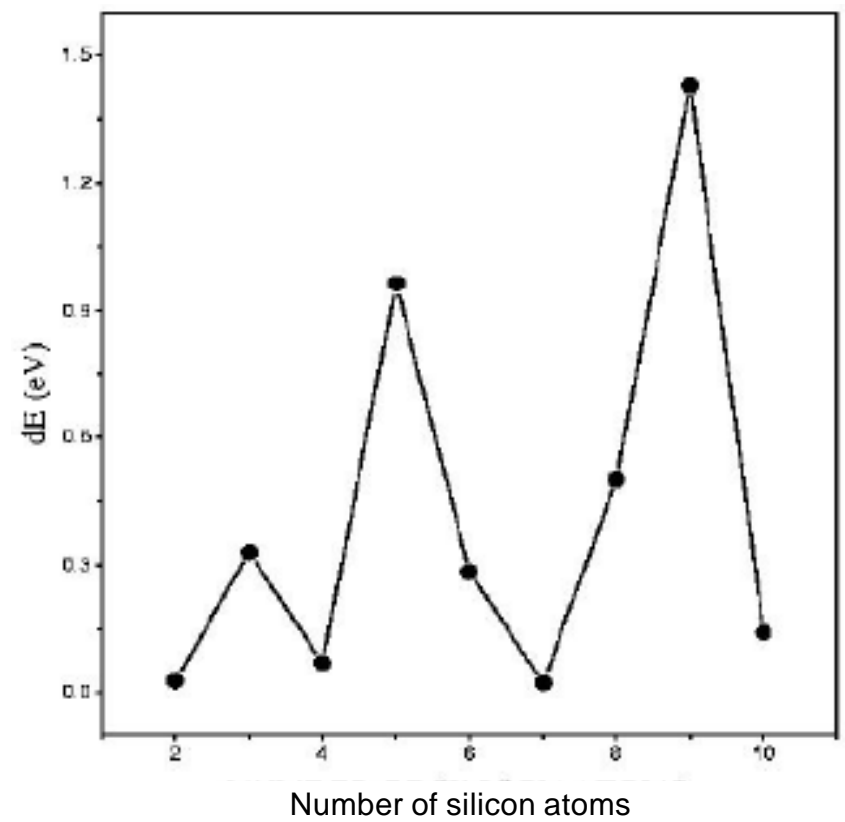

Figure 6. Difference, $\mathrm{d} E$, between total energy of the modified geometry of $\mathrm{Si}_{n} \mathrm{H}$, and the ground state energy of $\mathrm{Si}_{n}$ cluster vs number of silicon atoms in the clusters.

drawn by comparing $\mathrm{Si}_{n} \mathrm{H}$ and $\mathrm{Si}_{n}$ ground state geometries in $\$ 3$, as $\mathrm{Si}_{2}, \mathrm{Si}_{4}, \mathrm{Si}_{7}$ and $\mathrm{Si}_{10}$ clusters were least distorted by the addition of hydrogen. Thus the above discussion shows that, $\mathrm{Si}_{2}, \mathrm{Si}_{7}$ and $\mathrm{Si}_{10}$ clusters are the most stable clusters from both viewpoints of electronic as well as geometrical stability.

\section{Conclusions}

Results for the ground state structure and electronic properties of $\mathrm{Si}_{n} \mathrm{H}$ clusters have been presented using the Car-Parrinello molecular dynamics simulations. We find that hydrogen can form a bridge like $\mathrm{Si}-\mathrm{H}-\mathrm{Si}$ bond connecting two silicon atoms. Such bridge like bonds are thought to be present in $a-\mathrm{Si}: \mathrm{H}$ (Stutzmann et al 1985; Prasad and Shenoy 1996). Charge density calculations show that the $\mathrm{Si}-\mathrm{H}$ bond in all clusters is polar covalent. In clusters from $\mathrm{Si}_{7} \mathrm{H}$ to $\mathrm{Si}_{10} \mathrm{H}$, silicon atoms form a compact unit and hydrogen attaches to a silicon atom which is over-coordinated. Though hydrogen has small effect on the geometry of the host silicon cluster, it changes bond lengths and tries to distort the silicon cluster. This is similar to the behaviour of hydrogen in $a$-Si:H where it has been found that hydrogen creates local distortions as it moves (Prasad and Shenoy 1996). We find that hydrogen has a tendency to come out of compact silicon clusters and prefers to stay out of the cluster.

The first excitation electronic level gap of the $\mathrm{Si}_{n} \mathrm{H}$ clusters fluctuates as a function of size and this may provide a first principles basis for the short range potential fluctuations in $a-\mathrm{Si}: \mathrm{H}$ (Fritzche 1971; Agarwal 1996a, b; 
Sinha and Agarwal 2000). Our calculations show that the addition of hydrogen can cause large changes in the electronic structure of host $\mathrm{Si}_{n}$ cluster. Furthermore, it shows that $\mathrm{Si}_{2} \mathrm{H}, \mathrm{Si}_{3} \mathrm{H}, \mathrm{Si}_{5} \mathrm{H}, \mathrm{Si}_{7} \mathrm{H}, \mathrm{Si}_{9} \mathrm{H}$ and $\mathrm{Si}_{10} \mathrm{H}$ clusters are electronically more stable than $\mathrm{Si}_{4} \mathrm{H}, \mathrm{Si}_{6} \mathrm{H}$ and $\mathrm{Si}_{8} \mathrm{H}$ clusters. We find that $\mathrm{Si}_{2}, \mathrm{Si}_{4}, \mathrm{Si}_{7}$ and $\mathrm{Si}_{10}$ clusters are geometrically more stable than $\mathrm{Si}_{3}, \mathrm{Si}_{5}, \mathrm{Si}_{6}, \mathrm{Si}_{8}$ and $\mathrm{Si}_{9}$ clusters while $\mathrm{Si}_{2}, \mathrm{Si}_{5}, \mathrm{Si}_{6}, \mathrm{Si}_{7}$ and $\mathrm{Si}_{10}$ clusters are electronically more stable than $\mathrm{Si}_{3}, \mathrm{Si}_{4}, \mathrm{Si}_{8}$ and $\mathrm{Si}_{9}$ clusters.

\section{Acknowledgement}

This work was supported by the Department of Science and Technology, New Delhi via project No. SP/S2/M-51/96.

\section{References}

Agarwal S C 1996a Indian J. Pure \& Appl. Phys. 34597

Agarwal S C 1996b Bull. Mater. Sci. 1939

Balamurugan D and Prasad R 2001 Phys. Rev. B64 205406

Car R and Parrinello M 1985 Phys. Rev. Lett. 552471

Fritzche H 1971 J. Non-Cryst. Solids 649

Lu Z, Wang C and Ho K 2000 Phys. Rev. B61 2329

Prasad R and Shenoy S R 1996 Phys. Lett. A218 85

Sinha A K and Agarwal S C 2000 J. Vac. Sci. Technol. B18 1805

Stutzmann M, Jackson W B and Tsai C C 1985 Phys. Rev. B32 23 